\title{
Produção de conhecimento em política, planejamento e gestão na Revista Ciência \& Saúde Coletiva
}

\author{
Production of knowledge on policy, planning, and management in \\ Journal Ciência \& Saúde Coletiva
}

Cristiani Vieira Machado (https://orcid.org/0000-0002-9577-0301) ${ }^{1}$
Luciana Dias de Lima (https://orcid.org/0000-0002-0640-8387) ${ }^{1}$
Aylene Bousquat (http://orcid.org/0000-0003-2701-1570) ${ }^{2}$
Marcus Vinicius Pereira-Silva (https://orcid.org/0000-0002-4135-4011) ${ }^{3}$
Daniela Rangel Affonso Fernandes (https://orcid.org/0000-0002-0969-2707) ${ }^{4}$
Elizabeth Artmann (https://orcid.org/0000-0002-8690-5964) ${ }^{1}$
Ana Luiza D’Avila Viana (http://orcid.org/0000-0003-4498-899X) ${ }^{5}$
Sheyla Maria Lemos Lima (https://orcid.org/0000-0003-1450-0498) ${ }^{1}$

${ }^{1}$ Escola Nacional de Saúde Pública Sérgio Arouca, Fiocruz. R. Leopoldo Bulhões 1480, Manguinhos. 21041-210 Rio de Janeiro RJ Brasil.

cristiani.machado@ fiocruz.br

${ }^{2}$ Faculdade de Saúde Pública, Universidade de São Paulo (USP). São Paulo SP Brasil.

${ }^{3}$ Casa de Oswaldo Cruz e Observatório em Ciência, Tecnologia e Inovação em Saúde, Fiocruz. Rio de Janeiro RJ Brasil.

${ }^{4}$ Farmanguinhos, Fiocruz. Rio de Janeiro RJ Brasil. ${ }^{5}$ Faculdade de Medicina, USP. São Paulo SP Brasil.

\begin{abstract}
The area of Policy, Planning and Health Management (PPG) express the intersection between research, intervention and political action. The article analyzes the dynamics of knowledge production about PPG in the Journal Ciência \& Saúde Coletiva (CઐSC) from 1996 to 2019. The study articulates a bibliometric and qualitative approach to explore the profile of articles in three dimensions: thematic, methodological and authorship/institutional partnerships. 1680 PPG manuscripts were identified, corresponding to $28.3 \%$ of the total publications. Thematic analysis showed a strong influence of the context and the implementation of Unified Health System (SUS). Qualitative empirical studies predominated, followed by quantitative and quanti-qualitative studies. The combination of methodological procedures was frequent, with bibliographic review and interviews being the most cited. The average number of authors per article and national institutional partnerships increased. As most articles were published in Portuguese, the challenge of internationalization persists. The PPG area stands out in the Journal CerSC and in the production of scientific knowledge relevant to SUS.

Key words Health policy, planning, and management, Bibliometrics, Periodical publications
\end{abstract}

Resumo A área de Políticas, Planejamento $e$ Gestão em Saúde (PPG) é um dos pilares do campo da Saúde Coletiva, expressando a interseção entre pesquisa, intervenção e ação política. $O$ artigo analisa a dinâmica da produção de conhecimento em PPG, na revista Ciência \& Saúde Coletiva (C\&SC), de 1996 a 2019, articulando abordagem bibliométrica e qualitativa para caracterizar três dimensões: temática, metodológica e autoria/parcerias institucionais. Identificaramse 1.680 manuscritos, correspondendo a $28,3 \%$ do total de publicações da revista no período. A análise temática mostrou influência do contexto e da trajetória de implantação do Sistema Único de Saúde (SUS). Destacaram-se estudos empíricos qualitativos, seguidos dos quantitativos e dos com outros desenhos. A combinação de procedimentos metodológicos foi frequente, sendo mais citadas a revisão bibliográfica e entrevistas/questionários. $A$ maioria dos artigos foi publicada em português e, apesar do aumento das publicações em inglês no final do período, parcerias com autores de outros países foram raras, persistindo o desafio da internacionalização. Conclui-se que a área de PPG se destaca na revista CerSC, periódico que expressa o vigor do campo da Saúde Coletiva na produção de conhecimento científico relevante para o SUS e para a saúde da população.

Palavras-chave Politica, Planejamento e gestão em saúde, Bibliometria, Publicações periódicas 


\section{Introdução}

O campo da Saúde Coletiva se caracteriza pela forte interseção entre a pesquisa, a intervenção e a ação política, sendo definido como 'campo de saberes e práticas', que tem como objetivo central produzir conhecimentos visando à melhoria das condições de saúde e de vida da população.

A área de Políticas, Planejamento e Gestão em Saúde (PPG), reconhecida como um dos pilares constitutivos do campo (junto com a Epidemiologia e as Ciências Sociais em Saúde), tem tradição no desenvolvimento de estudos voltados à análise da dinâmica das políticas, dos sistemas e dos serviços de saúde, tendo a trajetória de sua produção, ao menos desde meados dos anos 1970, expressado forte influência dos processos políticos em saúde ${ }^{2}$.

A complexidade da área de PPG se relaciona com seu caráter interdisciplinar e com sua estreita vinculação com a Reforma Sanitária brasileira e o Sistema Único de Saúde (SUS). Os estudos em PPG apresentam grande diversidade temática e de objetos, buscando apoio em um amplo leque disciplinar e em distintos referenciais teóricos. A multiplicidade de perspectivas teórico-metodológicas e o imbricamento das pesquisas com as esferas da política e da ação ampliam as fronteiras da área, mas trazem desafios quanto ao rigor epistemológico e metodológico dos estudos ${ }^{3}$.

O periódico Ciência \& Saúde Coletiva (C\&SC), vinculado à Associação Brasileira de Saúde Coletiva (Abrasco), surgiu logo após a realização da $10^{\text {a }}$ Conferência Nacional de Saúde, e sua trajetória reflete tanto a institucionalização e a profissionalização da produção e divulgação científica do campo, como suas vinculações com os rumos da política e as transformações do sistema de saúde no Brasil ${ }^{4}$.

Tendo em vista que a revista C\&SC completa, em 2020, 25 anos de existência, é oportuno descrever e analisar a trajetória, a dinâmica e as características dos estudos publicados da área de PPG, considerando que a revista se constitui em um espaço privilegiado para ancorar os resultados de pesquisa, reflexões e debates sobre a saúde coletiva. É nesse contexto que este artigo se insere, ao analisar a produção da área de PPG expressa nos artigos publicados na revista Ciência \& Saúde Coletiva de 1996 a 2019, visando a contribuir para o conhecimento sobre as características dessa produção e para a compreensão da dinâmica da área de PPG no campo da Saúde Coletiva.

\section{Método}

Trata-se de estudo que articula abordagem bibliométrica e qualitativa ${ }^{5}$, pois aplicaram-se métodos estatísticos e matemáticos para explorar a trajetória dos artigos publicados sobre a área de PPG na revista C\&SC, no período de 1996 a 2019, associados à análise do perfil dos artigos segundo dimensões selecionadas.

Foram recuperados os artigos publicados em todas seções da revista desde 1996, ano de lançamento da revista, até 2019, no período de novembro de 2019 a fevereiro de 2020, utilizando a chave de busca 'planejamento OR gestão OR política OR gestión OR planificación' no campo título, resumo ou palavras-chaves. Os termos em espanhol foram necessários, visto que os artigos submetidos nesse idioma não possuem a obrigatoriedade de terem resumos, títulos e palavras-chave em português. Não foi incluído o termo 'saúde' e não foram adotados critérios de exclusão, uma vez que se considerou que todos os artigos recuperados abordam diretamente ou tangenciam a área de pesquisa analisada.

Utilizaram-se duas formas de recuperação, uma através da coleta manual na página da revista na SciELO (https://www.scielosp.org/journal/ $\csc /)(\mathrm{n}=1.153)$ e outra automática por meio da ferramenta MAURO (https://mauro.icict.fiocruz.br/) $(\mathrm{n}=1.788)$, desenvolvida para capturar e exportar metadados das revistas indexadas na SciELO. Cabe destacar que todos os números da C\&SC estão disponíveis nessa base.

Inicialmente foram geradas duas planilhas distintas utilizando o software Microsoft Excel, uma para os dados recuperados manualmente e outra através do MAURO. Apesar de utilizar a mesma estratégia de busca, os resultados foram distintos, indicando, assim, limites na recuperação da informação em ambos procedimentos de coleta. Após a combinação das duas planilhas e a exclusão de duplicatas, chegou-se ao total de 1.680 artigos a serem analisados.

Definiram-se as seguintes dimensões de análise dos artigos selecionados: principais temas abordados, métodos utilizados, seção da revista em que o artigo foi publicado, idioma de publicação, autoria e parcerias estabelecidas. Para cada dimensão, utilizaram-se procedimentos específicos.

Para auxiliar no mapeamento e na análise dos principais temas abordados, foi utilizada uma técnica de visualização de similaridade através do software VOSviewer ${ }^{6}$, usando a medida de "força de associação" . Considerando os títulos 
e os resumos em inglês, dois termos são considerados fortemente relacionados se ocorrem frequentemente nos artigos. Nos mapas, cada termo é representado por um círculo e, quanto mais próximo eles estiverem posicionados, mais relacionados são. $\mathrm{O}$ diâmetro e o tamanho dos rótulos indicam o número de ocorrências do termo. Foi utilizado o método de contagem binário, ou seja, a presença de um determinado termo é considerada somente uma única vez, sendo que o número mínimo de ocorrências de um termo para análise é de cinco e foram selecionados $60 \%$ dos termos mais relevantes. $\mathrm{O}$ número mínimo de termos para formação de agrupamentos (clusters) foi de $25 \%$ do total de termos.

Para a classificação metodológica dos trabalhos, foram extraídas e padronizadas informações referentes aos desenhos/tipos de estudo e procedimentos metodológicos utilizados. As seguintes classes foram definidas para os desenhos/ tipos de estudo: 1) estudos empíricos com ênfase quantitativa, qualitativa e quanti-qualitativo; 2) estudos de revisões bibliográficas; 3 ) estudos teórico-conceituais; 4) estudos com outro desenho; 5) não se aplica, incluídos artigos para os quais não se identificou um desenho de estudo. Estudos de caso, análises históricas, análises de conjuntura, estudos comparados e relatos de intervenções (criação de métodos, de painel de indicadores, etc.) foram classificados como estudos empíricos (qualitativo, quantitativo ou quanti-qualitativo). Em estudos com outro desenho foram considerados os relatos de experiência, os ensaios e as análises de política não configuradas como estudos empíricos. Estudos teórico-conceituais incluíram apenas os artigos com uma discussão explícita sobre conceitos ou os que apresentavam uma teoria explicativa. Cada artigo só foi classificado em uma categoria.

Como procedimentos metodológicos foram considerados: análise de dados secundários, análise documental, revisão bibliográfica, entrevista ou questionário individual, método coletivo (entrevista coletiva, grupo focal, reunião), observação (participante ou não, diário de campo, etc.), outro procedimento e, por fim, a categoria não se aplica. Um mesmo artigo pode ter tido vários procedimentos metodológicos.

Complementarmente identificaram-se as seções da revista nas quais os estudos foram publicados: Editorial, Artigo Temático, Temas Livres, Revisão, Opinião, Resenha, Debate, Carta, Debatedores (resposta ao artigo publicado na seção Debate), Destaque, Construtores (relato biográfico sobre profissionais de referência no campo da
Saúde Coletiva) e Press release (síntese informativa de artigo publicado).

O total de artigos da área de PPG foi comparado ao total de artigos publicados na revista C\&SC, recuperados manualmente na página da revista na SciELO, considerando-se todos os artigos publicados em todas seções da revista acima discriminados.

Para caracterização da autoria e parcerias institucionais, foram consideradas a instituição e país do $1^{\circ}$ autor, o número total de autores, o número de instituições envolvidas e se estas incluíam mais de um estado da federação e/ou país. Foram também considerados os idiomas em que os artigos foram veiculados.

Todas as análises se referem ao período total do estudo (1996 a 2019) e à comparação entre três períodos arbitrários de 8 anos, a saber: 1996 2003, 2004-2011 e 2012-2019.

\section{Resultados e discussão}

Foram recuperados 1.680 manuscritos da área de PPG publicados na revista C\&SC, correspondendo a $28,3 \%$ do total de publicações do período de 1996 a 2019. Observou-se aumento da participação proporcional de artigos da área de PPG, que representava 23,1\% $(\mathrm{n}=110)$ entre 1996 e 2003, passando para 29,3\% ( $\mathrm{n}=981)$ entre 2012 e 2019 (Tabela 1).

$\mathrm{O}$ aumento do número de artigos publicados de PPG ao longo dos anos, em parte, pode ser explicado pela mudança de periodicidade de publicação da revista, que até 1999 era semestral, de 2000 a 2006, quadrimestral, de 2007 a 2010, bimestral e a partir de 2011, mensal. Outros fatores explicativos seriam o crescimento do número de pesquisas ${ }^{8}$; o aumento do número de programas de pós-graduação e o sistema de avaliação de pósgraduação privilegiar a produção científica como indicador de qualidade ${ }^{9}$ e a própria dinâmica da implementação do Sistema Único de Saúde ${ }^{10}$.

\section{Análise temática}

A análise temática dos artigos no período mostrou a conformação de agrupamentos de termos que apresentaram variações ao longo do período (Figura 1).

No conjunto do período de 1996 a 2019, a análise temática envolveu 534 termos que foram reunidos em dois agrupamentos principais: 'políticas e sistemas de saúde’ e 'agravos, doenças e populações específicas' (Figura 1a). 
Tabela 1. Artigos sobre política, planejamento e gestão em saúde publicados na Revista Ciência \& Saúde Coletiva segundo desenhos de estudo no período de 1996 a 2019.

\begin{tabular}{|c|c|c|c|c|c|c|c|c|}
\hline \multirow{3}{*}{ Desenho/Tipo de Estudo } & \multicolumn{6}{|c|}{ Períodos } & \multirow{2}{*}{\multicolumn{2}{|c|}{$\begin{array}{c}\text { Total } \\
1996 \text { a } 2019 \\
\end{array}$}} \\
\hline & \multicolumn{2}{|c|}{1996 a 2003} & \multicolumn{2}{|c|}{2004 a 2011} & \multicolumn{2}{|c|}{2012 a 2019} & & \\
\hline & $\mathbf{N}$ & $\%$ & $\mathbf{N}$ & $\%$ & $\mathbf{N}$ & $\%$ & $\mathbf{N}$ & $\%$ \\
\hline Estudo empírico com ênfase qualitativa & 17 & 15,4 & 212 & 36,1 & 303 & 30,9 & 532 & 31,7 \\
\hline Estudo empírico com ênfase quantitativa & 17 & 15,5 & 86 & 14,6 & 305 & 31,1 & 408 & 24,3 \\
\hline $\begin{array}{l}\text { Outros desenhos (ensaio, análise de } \\
\text { política e relato de experiência) }\end{array}$ & 30 & 27,3 & 109 & 18,5 & 98 & 10,0 & 237 & 14,1 \\
\hline $\begin{array}{l}\text { Estudo de revisões (narrativa, integrativa } \\
\text { e sistemática) }\end{array}$ & 26 & 23,6 & 45 & 7,6 & 95 & 9,7 & 166 & 9,9 \\
\hline Não se aplica & 9 & 8,2 & 62 & 10,5 & 69 & 7,0 & 140 & 8,3 \\
\hline Estudo empírico quanti- qualitativo & 7 & 6,4 & 46 & 7,8 & 76 & 7,7 & 129 & 7,7 \\
\hline Estudo teórico-conceitual & 4 & 3,6 & 29 & 4,9 & 35 & 3,6 & 68 & 4,0 \\
\hline Total de publicações PPG & 110 & 100,0 & 589 & 100,0 & 981 & 100,0 & 1680 & 100,0 \\
\hline $\begin{array}{l}\text { \% de publicações PPG no total de } \\
\text { publicações da revista }\end{array}$ & \multicolumn{2}{|c|}{23,1} & \multicolumn{2}{|c|}{27,8} & \multicolumn{2}{|c|}{29,3} & \multicolumn{2}{|c|}{28,3} \\
\hline
\end{tabular}

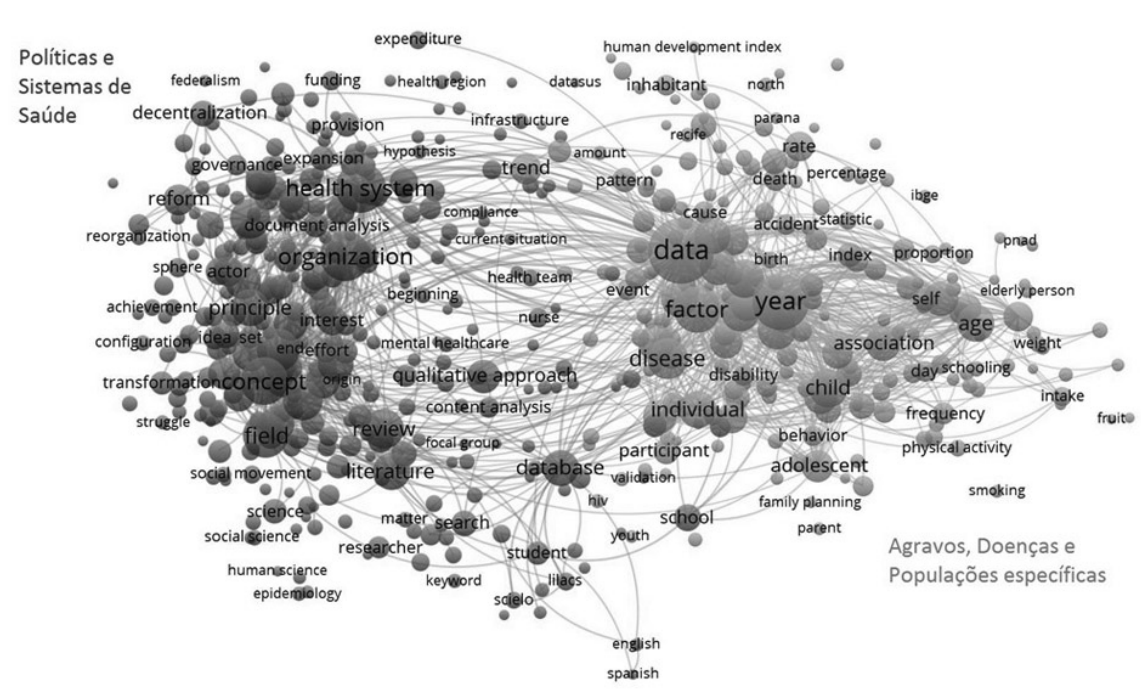

Figura 1a. Agrupamentos temáticos identificados nos artigos de Políticas, Planejamento e Gestão publicados na revista Ciência \& Saúde Coletiva, de 1996 a 2019.

Fonte: Dados da pesquisa, construída a partir do SciELO.

No primeiro, que agrega o maior número de termos (320), os temas de maior frequência e que nucleiam a relação com os demais são: sistema de saúde, Sistema Único de Saúde e organização. Também há nesse agrupamento um conjunto de termos referentes à construção e configuração das políticas e do campo, reunindo expressões como: conceito, princípios, constituição, reflexão, debate, construção, campo, contribuição, propostas, papel e sociedade. Há ainda outros termos relacionados à gestão do sistema, que incluem expressões como: governo, governança, política social, descentralização, regionalização e coordenação. Por fim, há um conjunto de termos, conectados aos demais, que indica a forte presença de estudos de revisão entre as publicações da revista, tais como: revisão, literatura, base de dados, busca e publicação. 
O segundo agrupamento, que reúne 214 termos, compreende expressões relacionadas a determinados agravos e doenças, tais como violência, seguido por outros como alimentação e nutrição, tabagismo, alcoolismo, hipertensão, diabetes. Entre os grupos populacionais específicos, foram identificados com maior destaque os termos mulher e criança, mas apareceram também os temas adolescente, idoso, homem. Um conjunto de termos expressivo é de caráter mais metodológico, incluindo palavras como dados, sistema de informações, ano, aumento, prevalência, características, fator, variável, questionário, survey, número. Esse conjunto está muito próximo a termos como mortalidade, hospitalizações, sugerindo estudos epidemiológicos e relacionados ao uso de serviços de saúde.

O período de 1996 a 2003 compreende os oito primeiros anos da revista C\&SC. A análise temática desse período mostrou três agrupamentos fortemente relacionados entre si: 'SUS e determinantes sociais', 'campo da Saúde Coletiva' e 'implantação e gestão do SUS' (Figura 1b). No primeiro deles, com 37 termos, destacam-se as expressões Sistema Único de Saúde, planejamento, proposta, e aspectos relacionados aos determinantes sociais da saúde, tais como: desigualdade, equidade, condição, ambiente, população, espaço, trabalhador, sociedade civil, morbidade e violência. O segundo agrupamento, com 23 termos, expressa reflexões sobre a constituição do campo da Saúde Coletiva, tais como: saúde pública, campo, perspectiva, instituição, pesquisa, ciência, conhecimento, publicação, educação, estabelecimento. $\mathrm{O}$ terceiro agrupamento inclui 21 termos fortemente relacionados com a implantação inicial e a gestão do SUS: política de saúde, política pública, implementação, descentralização, município, governo, financiamento.

Destacam-se em síntese, os temas relacionados à reflexão sobre o campo da Saúde Coletiva, os determinantes do processo saúde-doença e os desafios que sobressaíram na primeira década de implantação do SUS, como a descentralização e o financiamento.

No período de 2004 a 2011, identificaram-se dois agrupamentos temáticos: 'Saúde Coletiva, sistema e serviços de saúde' e 'agravos, doenças e populações específicas' (Figura 1c). O primeiro agrupamento compreende 223 termos, que expressam questões estratégicas relativas à implementação do SUS, com maior destaque para os serviços de saúde, tais como: sistema de saúde, serviços de saúde, atenção à saúde, atenção primária à saúde. Temas relativos às políticas de saúde e gestão do SUS também se mantém, como: descentralização, reforma, política de saúde, constituição, financiamento, sustentabilidade. Ainda

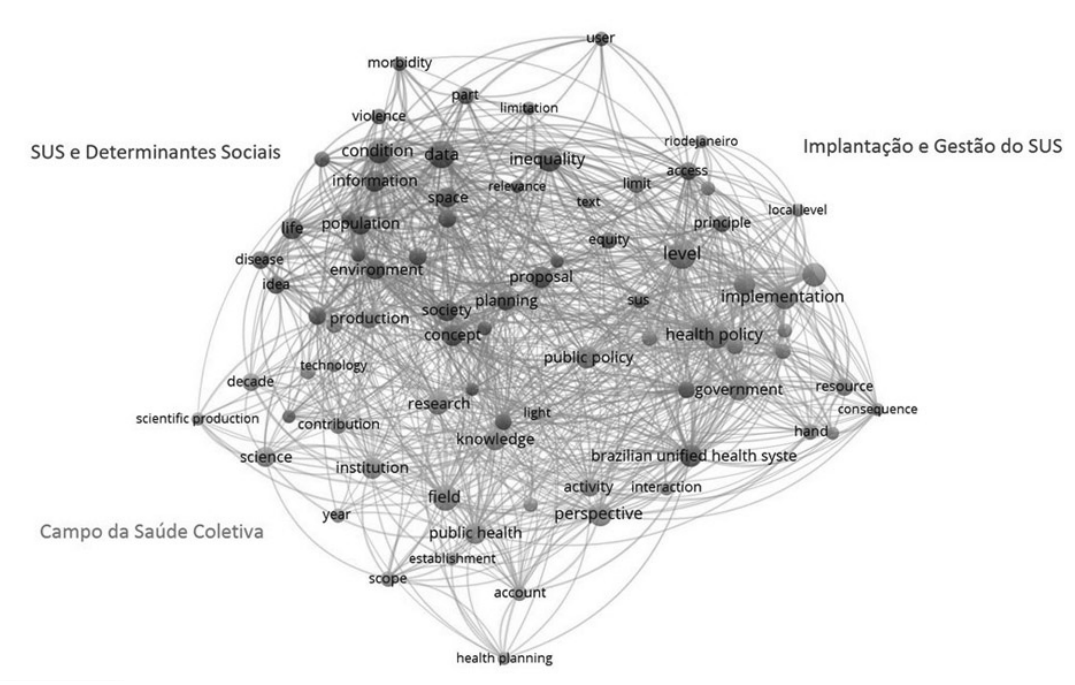

Figura 1b. Agrupamentos temáticos identificados nos artigos de Políticas, Planejamento e Gestão publicados na revista Ciência \& Saúde Coletiva, de 1996 a 2003. 


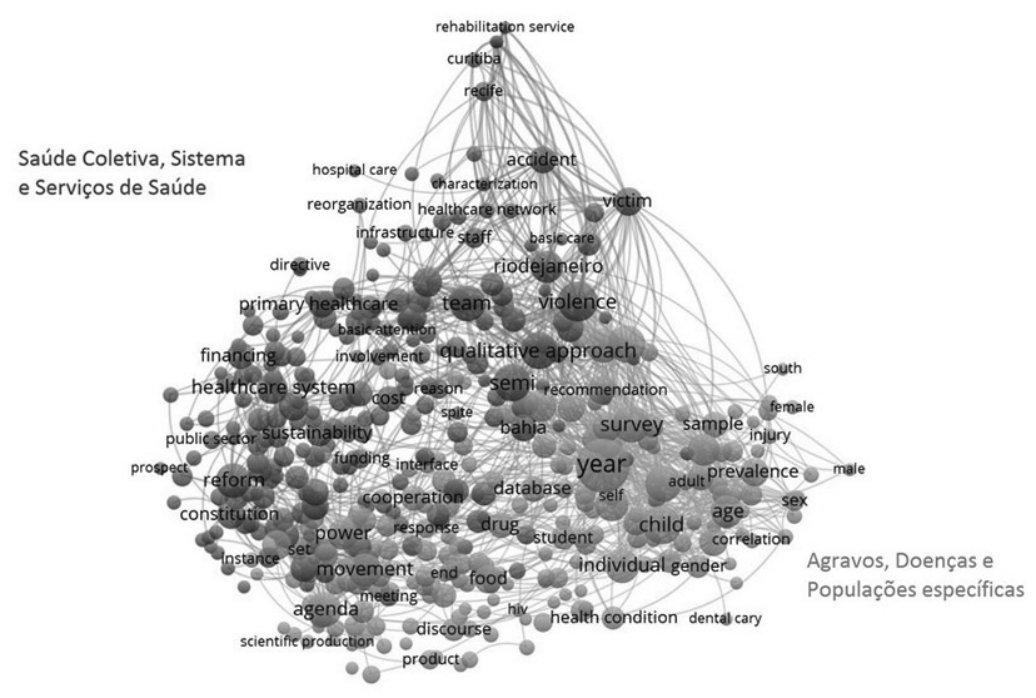

Figura 1c. Agrupamentos temáticos identificados nos artigos de Políticas, Planejamento e Gestão publicados na revista Ciência \& Saúde Coletiva, de 2004 a 2011; (1d) 2012 a 2019.

Fonte: Dados da pesquisa, construída a partir do SciELO.

nesse agrupamento, ressalte-se o tema da violência, que se conecta fortemente também com temas do segundo agrupamento. O segundo agrupamento inclui 208 termos e se diferencia por reunir temas relacionados a agravos, doenças e populações específicas: mulheres, adolescentes, crianças, condições de saúde, alcoolismo, nutrição.

O último período, de 2012 a 2019, reflete o momento mais recente de implantação do SUS, e expressa dois agrupamentos com características temáticas semelhantes ao momento anterior: 'sistemas e serviços de saúde' e 'agravos, doenças e populações específicas' (Figura 1d). Entretanto, percebe-se menor conexão entre ambos. $\mathrm{O}$ primeiro agrupamento reúne 372 termos, fortemente relacionados à organização e gestão dos sistemas e serviços de saúde. Novos temas se destacam - regionalização, sustentabilidade, participação social, governança, accountability enquanto outros se mantém - equipes, atenção primária e serviços de saúde. O segundo agrupamento agrega 315 termos, sendo que crianças e adolescentes ganham maior visibilidade, assim como determinados tipos de violência (violência no trânsito, violência sexual e violência física) e distúrbios alimentares (obesidade).

Assim, no $2^{\circ}$ período (2004 a 2011), políticas de saúde prioritárias na agenda nacional da saú- de, como a atenção básica e a estratégia de Saúde da Família ${ }^{11}$ foram objetos frequentes de estudo de pesquisadores do campo. Tais temas se mantém no último período analisado, acrescidos de outros que ganham destaque na política nacional, como a regionalização em saúde ${ }^{12}$, reiterando a conexão da produção da área com o processo de implantação do SUS.

No que concerne a problemas de saúde ou a grupos populacionais específicos, em todo o período chama a atenção o destaque conferido aos temas da violência e à saúde da mulher ou da criança; em alguns momentos, o tema da alimentação e nutrição também ganha espaço. Por outro lado, temas clássicos de saúde pública como doenças infecciosas ou crônico-degenerativas, embora presentes, não se sobressaem de forma expressiva entre os artigos da área de PPG.

Do ponto de vista temático, observa-se forte influência do contexto das políticas dos diversos momentos da trajetória de implantação do SUS nos objetos de estudo priorizados nos artigos da área de PPG.

\section{Caracterização dos métodos}

No período completo, os tipos de desenho que mais se destacaram foram os estudos empí- 


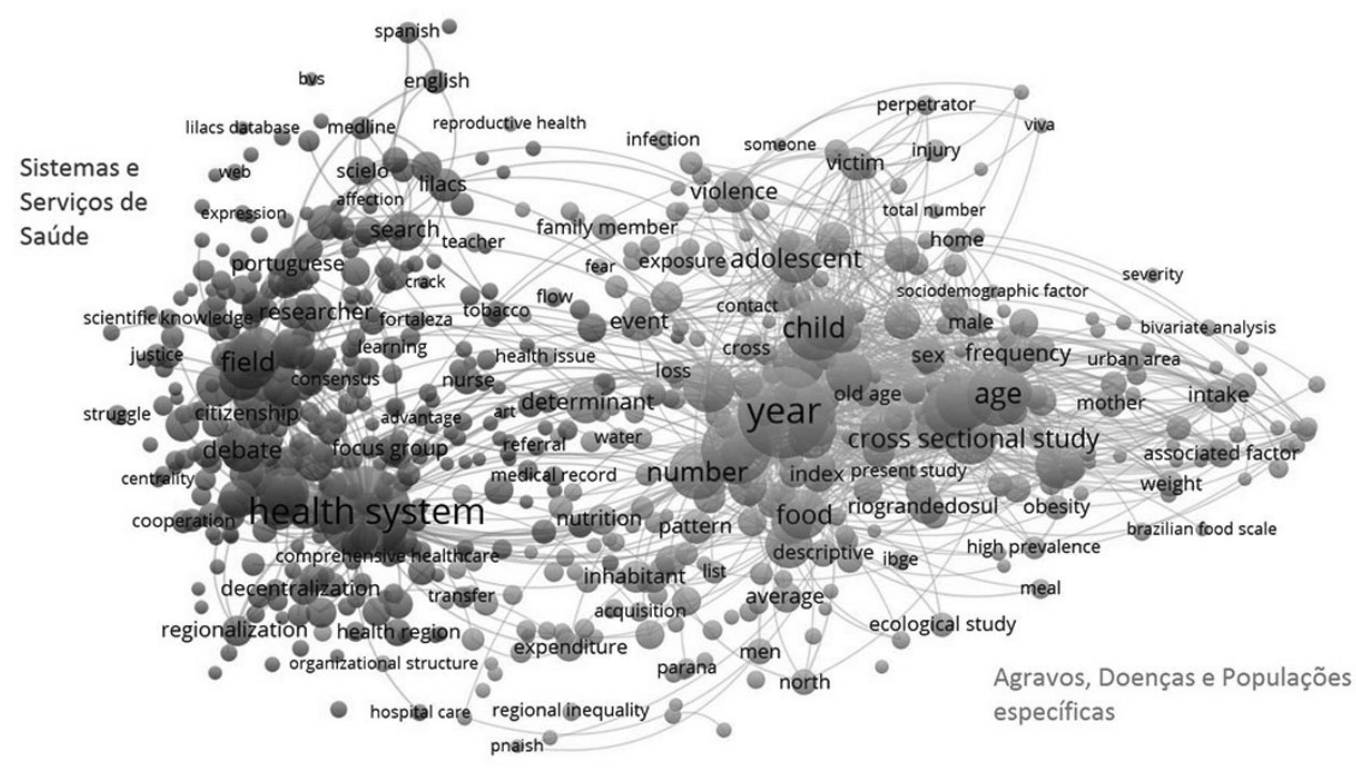

Figura 1d. Agrupamentos temáticos identificados nos artigos de Políticas, Planejamento e Gestão publicados na revista Ciência \& Saúde Coletiva, de 2012 a 2019.

Fonte: Dados da pesquisa, construída a partir do SciELO.

ricos com ênfase qualitativa $(31,7 \%)$ e quantitativa $(24,3 \%)$, seguidos pelos classificados como outros desenhos (14,1\%). Ainda se observa que os estudos empíricos quanti-qualitativos totalizaram 129 manuscritos, representando 7,7\% do total. Todos os estudos empíricos (1072) representaram $63,7 \%$ dos manuscritos publicados no período (Tabela 1).

No primeiro período (1996 a 2003) predominaram outros estudos $(27,3 \%)$, estudos de revisão $(23,6 \%)$ e por fim, estudos empíricos com ênfase quantitativa $(15,5 \%)$ e qualitativa $(15,4 \%)$ (Tabela 1).

No período de 2004 a 2011 se destacaram os estudos empíricos com ênfase qualitativa $(36,1 \%)$, os outros estudos $(18,5 \%)$ e os estudos empíricos com ênfase quantitativa $(14,6 \%)(\mathrm{Ta}-$ bela 1).

No último período (2012 a 2019) tiveram proeminência os estudos empíricos com ênfase quantitativa $(31,1 \%)$ e qualitativa $(30,9 \%)$, seguidos dos outros estudos $(10,0 \%)$ das revisões $(9,7 \%)$ e dos estudos quanti-qualitativos $(7,7 \%)$ (Tabela 1).

Destaca-se a relevância de outros desenhos nos dois primeiros períodos, em especial no período de 1996 a 2003, enquanto no período mais recente, ganham importância os estudos empíricos. Em outros estudos (237), considerando todo o período, predominam os ensaios (146), seguidos de relatos de experiência (51) e das análises de política (40).

Registram-se no período estudado, 140 estudos não classificados em nenhum tipo de desenho (não se aplica) (Tabela 1). Aqui estão incluídos a maior parte dos artigos publicados na seção opinião (42), a totalidade dos artigos publicados nas seções debatedores (44), editorial (21), resenha (16), construtores (8), press release (5) e carta (1).

Quanto aos procedimentos metodológicos, considerando o período completo de 1996 a 2019 , observa-se que o mais utilizado foi a revisão bibliográfica (758), seguido das entrevistas/questionários individuais (625), da análise documental (424) e da análise de dados secundários (358). Por fim, foram referidos a observação direta (163) e os métodos coletivos (136) (Tabela 2).

As entrevistas/ questionários individuais foram os mais referidos nos estudos empíricos qualitativos (312), quantitativos (222) e quantiqualitativos (81). As observações diretas (129) e os métodos coletivos (96) foram majoritariamente referidos nos estudos empíricos qualitativos, embora também sejam citadas nos estu- 
Tabela 2. Artigos sobre política, planejamento e gestão em saúde publicados na Revista Ciência \& Saúde Coletiva segundo desenhos de estudo e procedimentos metodológicos de 1996 a 2019.

\begin{tabular}{|c|c|c|c|c|c|c|c|c|}
\hline \multirow[b]{2}{*}{$\begin{array}{c}\text { Desenho/Tipo de } \\
\text { Estudo }\end{array}$} & \multicolumn{8}{|c|}{ Procedimento Metodológico } \\
\hline & $\begin{array}{c}\text { Análise } \\
\text { de dados } \\
\text { secundários }\end{array}$ & $\begin{array}{c}\text { Análise } \\
\text { documental }\end{array}$ & $\begin{array}{c}\text { Revisão } \\
\text { bibliográfica }\end{array}$ & $\begin{array}{c}\text { Entrevistas ou } \\
\text { Questionários } \\
\text { individuais }\end{array}$ & $\begin{array}{l}\text { Métodos } \\
\text { coletivos }\end{array}$ & Observação & Outros & $\begin{array}{l}\text { Não se } \\
\text { aplica }\end{array}$ \\
\hline $\begin{array}{l}\text { Estudo empírico } \\
\text { com ênfase } \\
\text { qualitativa }\end{array}$ & 46 & 235 & 188 & 312 & 96 & 129 & 14 & 0 \\
\hline $\begin{array}{l}\text { Estudo empírico } \\
\text { com ênfase } \\
\text { quantitativa }\end{array}$ & 205 & 40 & 88 & 222 & 5 & 9 & 38 & 0 \\
\hline $\begin{array}{l}\text { Estudo empírico } \\
\text { quanti-qualitativo }\end{array}$ & 72 & 63 & 50 & 81 & 16 & 15 & 4 & 0 \\
\hline $\begin{array}{l}\text { Estudo de revisões } \\
\text { (narrativa, } \\
\text { integrativa e } \\
\text { sistemática) }\end{array}$ & 5 & 8 & 166 & 0 & 0 & 0 & 2 & 0 \\
\hline $\begin{array}{l}\text { Estudo teórico- } \\
\text { conceitual }\end{array}$ & 2 & 9 & 68 & 0 & 0 & 0 & 0 & 0 \\
\hline $\begin{array}{l}\text { Outros desenhos } \\
\text { (ensaios, análise de } \\
\text { políticas e relatos de } \\
\text { experiência) }\end{array}$ & 28 & 69 & 198 & 10 & 19 & 10 & 13 & 1 \\
\hline Não se aplica & 0 & 0 & 0 & 0 & 0 & 0 & 0 & 140 \\
\hline Total & 358 & 424 & 758 & 625 & 136 & 163 & 71 & 141 \\
\hline
\end{tabular}

dos empíricos quanti-qualitativos, nos outros estudos e ainda em poucos estudos empíricos quantitativos (Tabela 2). Já a revisão bibliográfica, coerentemente com o esperado, se destacou nos estudos de revisão, teórico-conceituais e com outros desenhos.

Foram ainda relatados outros procedimentos metodológicos, dentre eles, exames clínicos, nutricionais, antropométricos e análise laboratorial (Tabela 2).

Segundo as seções de publicação da revista, considerando o período de 1996 a 2019, predominaram os artigos temáticos (998), seguidos pelos temas livres (395) e artigos de revisão (81). No período de 1996-2003, se destacaram numericamente os artigos das seções artigos temáticos (82); os demais se distribuíram nas seções temas livres (13), opinião (8) e debate (7). A predominância dos artigos temáticos e de temas livres foi mantida nos períodos seguintes. De 2004 a 2001, foram 343 artigos temáticos e 135 de temas livres e de 2012 a 2019, 573 temáticos e 247 temas livres. Porém, nos 2 últimos períodos, observa-se maior distribuição nas demais seções de publicação, com destaque para debate, debatedores e opinião. Crescem comparativamente os artigos publicados na seção revisão.

Por fim, observa-se que nos artigos temáticos destacaram-se os estudos empíricos com ênfase qualitativa (359), quantitativa (259) e quantiqualitativa (86). Comportamento semelhante apresentaram os artigos de temas livres, com uso de estudos empíricos com ênfase qualitativa (152), quantitativa (142) e quanti-qualitativa (35). São relevantes também os outros desenhos (185) que incluem os ensaios, as análises de política e relatos de experiência. Dos 60 artigos publicados na seção debate, 23 foram identificados na categoria outros desenhos e 12 como estudos empíricos com ênfase qualitativa. Para apenas três não se identificou algum tipo de desenho de estudo. Na seção destaque foram publicados apenas dois manuscritos, sendo um estudo empírico com ênfase qualitativa e outro classificado como outros desenhos. Nas demais seções - debatedores, editorial, resenha, construtores e press release - como esperado, não foi identificado nenhum desenho de estudo.

Em linhas gerais, os artigos parecem ganhar maior estruturação ao longo do tempo, talvez 
expressando um amadurecimento metodológico na área. O predomínio de estudos empíricos de tipo qualitativo era de certa forma esperado, mas também foi expressiva a presença de artigos com abordagens quantitativas ou que articulam métodos qualitativos e quantitativos. Em consequência, a combinação de técnicas de pesquisa também é frequente, embora se destaquem a análise documental e a realização de entrevistas individuais nos estudos empíricos analisados.

Por outro lado, a revista publicou no período muitos artigos em forma de ensaios, análises de conjuntura política e relatos de experiência, não estruturados como artigos de pesquisa original. Isso pode ser em parte explicado pela vinculação estreita da área da PPG com a dinâmica de implementação do SUS e a radicalidade com que essa área expressa a mistura entre "saberes e práticas", constitutiva do campo da Saúde Coletiva ${ }^{13,14}$. Associe-se a isso a orientação da política editorial da revista no que concerne às seções disponíveis para apresentação de artigos e à prática de organização de números temáticos voltados a questões estratégicas do SUS, muitos dos quais conjugam chamadas públicas com convites a especialistas do campo da Saúde Coletiva. No que concerne à área de PPG, parte expressiva dos autores associam formação ou atividade acadêmica com atuação em gestão ou serviços de saúde, com uma fluidez que se reflete não apenas nos temas, mas também nos métodos dos estudos e nos tipos de artigos publicados.

\section{Caracterização da autoria e parcerias institucionais}

A média de número de autores por artigo foi de 3,3 com mediana de 3,0 e desvio padrão de 2,0 . No entanto, estes valores variam nos períodos analisados. No primeiro período, a média foi de 2,0 autores, no segundo, foi de 2,8 e no último foi de 3,8. No Gráfico 1 visualiza-se esta tendência de crescimento do número de autores.

A proporção de artigos com autores de mais de uma instituição passou de $33,6 \%$ no primeiro período analisado para 59,6\% a partir de 2012 . No entanto, a colaboração entre instituições de mais de uma unidade da federação ainda é tímida, respondendo por $28,4 \%$ dos artigos do último período analisado e $23,8 \%$ do total. A colaboração entre pesquisadores sediados em diferentes países foi de apenas 2,8\% nos 24 anos da C\&SC.

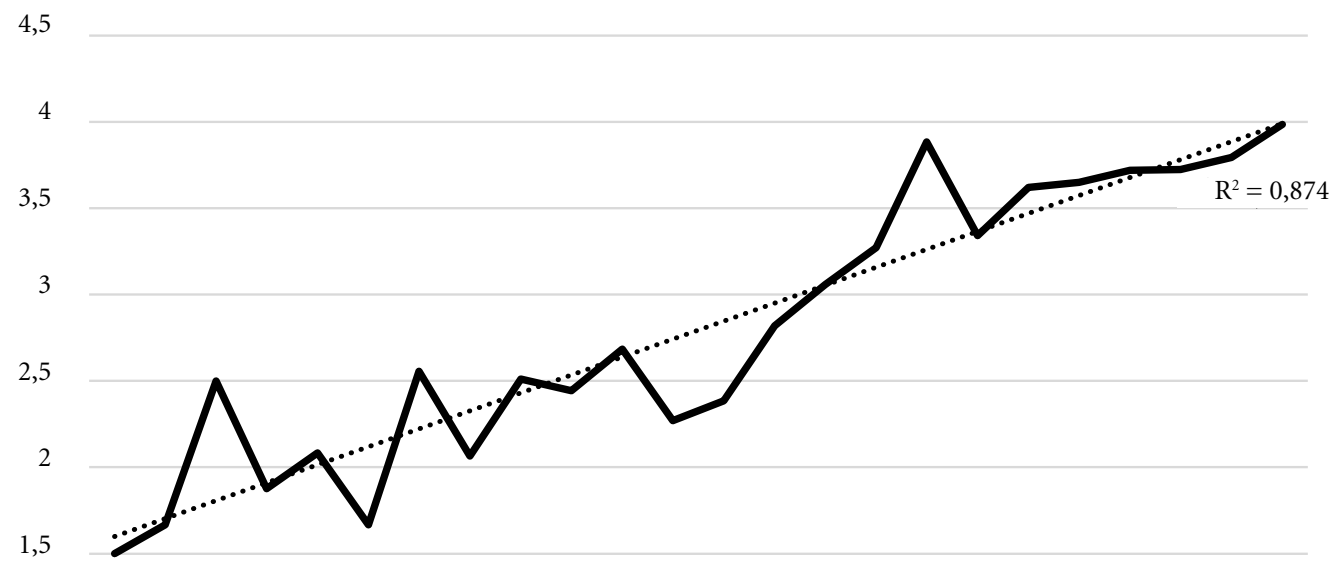

0,5

0

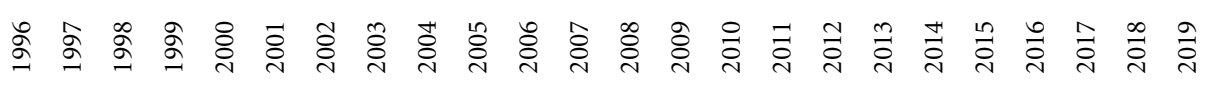

Gráfico 1. Número médio de autores por artigo Área de Política, Planejamento e Gestão- C\&CS 1996-2019. 
A maioria dos primeiros autores são mulheres $(64,3 \%)$, com crescimento constante deste percentual ao longo dos períodos. Quanto às instituições dos primeiros autores, a Fiocruz, considerando todas as suas unidades nos diferentes estados brasileiros, responde sozinha por $23,2 \%$ do conjunto dos artigos, valendo ressaltar que entre 1996 a 2003 este percentual era de 42,7\%. Em seguida, destacam-se os autores afiliados à USP (6,8\%); seguidos pela UERJ, Unicamp; UNB, UFBA, UFRJ e UFMG $(3,7 \% ; 3,5 \% ; 3,4 \% ; 3,2 \%$; $3,1 \%$ e $2,8 \%$ respectivamente). Chama atenção que $7,7 \%$ dos artigos são de pesquisadores vinculados a diferentes esferas administrativas do SUS ou a serviços de saúde.

Quanto ao idioma, apenas 90 artigos $(5,4 \%)$ não foram publicados em português. Observa-se importante crescimento da publicação em inglês no último período analisado com 50,3\% (493) veiculados neste idioma, quando o mesmo dado é analisado para todos os anos este percentual cai para $31,5 \%$. Apenas 58 artigos foram publicados em espanhol e 5 em francês. Considerando as instituições dos primeiros autores, o periódico veicula fundamentalmente autores brasileiros $(94,0 \%)$, seguidos de portugueses $(1,2 \%)$, argentinos $(0,8 \%)$, canadenses $(0,7 \%)$ e estadunidenses $(0,7 \%)$.

Destaca-se o crescimento do número de autores e, por consequência, de instituições envolvidas na produção analisada, sendo tímida a parceria entre instituições de mais de uma unidade da federação e mais ainda entre autores/instituições de diferentes países. Esse quadro pode estar associado à participação predominante de autores vinculados a Fiocruz e também ao baixo percentual de estudos publicados em outro idioma, que não o português, durante o período estudado. Ressalta-se que, no último período analisado, já se verifica o esforço que a revista vem fazendo para alcançar penetração internacional, tendo em vista que metade dos artigos foram publicados na língua inglesa.

\section{Conclusões}

A área de PPG teve marcante presença nas publicações da revista C\&SC no período estudado, com participação crescente de artigos em termos absolutos e relativos. O perfil dos artigos em PPG expressa as políticas editoriais da revista, mas também dinâmica de produção do conhecimento no campo da Saúde Coletiva e a relação da área com o contexto e a dinâmica das políticas e do SUS, que se manifesta na multiplicidade dos temas dos estudos em cada momento e na sua variação ao longo do tempo. Se no primeiro período se destacam reflexões sobre a constituição do campo da Saúde Coletiva e do SUS, no último sobressaem temas relacionados à implementação do SUS, em estreita relação com serviços, agravos e grupos específicos.

A diversidade metodológica da área se refletiu nos artigos publicados, observando-se indícios de certo adensamento metodológico ao longo do período. Por outro lado, a revista manteve artigos com formato flexível - como ensaios, opinião, debate - buscando abarcar a fluidez das reflexões produzidas no campo da Saúde Coletiva e, em especial, na área da PPG.

A revista C\&SC tem investido na internacionalização, contudo, cenários desfavoráveis de apoio e financiamento não permitiram uma velocidade maior no alcance deste intuito. Assim, ainda se constitui um desafio para a revista incrementar a publicação de artigos que expressem maior parceria interinstitucionais nacionais e internacionais.

Um limite deste estudo foi a sua delimitação a um periódico científico. Sugere-se a realização de análises futuras das publicações em PPG em perspectiva comparada entre revistas do campo em âmbito nacional e internacional, bem como a atualização de análises abrangentes referentes ao conjunto da produção da área em PPG, considerando artigos e livros científicos. Tal esforço é importante para a compreensão das contribuições dessa área para o campo da Saúde Coletiva e a compreensão das transformações nos sistemas de saúde, especialmente em tempos em que a ciência e as políticas sociais passam por questionamentos e restrições de várias ordens. Nesse contexto, a revista C\&SC continuará sendo um dos principais bastiões da defesa da ciência e de expressão do campo da Saúde Coletiva na produção de conhecimento socialmente relevante. 


\section{Colaboradores}

CV Machado, LD Lima, A Bousquat, MV PereiraSilva, DRA Fernandes, E Artmann, ALD Viana e SML Lima trabalharam na concepção, no levantamento e análise das informações, na elaboração e revisão do manuscrito.

\section{Referências}

1. Paim J, Almeida Filho N. A crise da saúde pública e a utopia da saúde coletiva. Salvador: Casa da Qualidade Editora; 2000

2. Teixeira CF. Produção Científica na Área de Política, Planejamento e Gestão em Saúde 1975-2010: temas e teorias. In: Baptista TWF, Azevedo CS, Machado CV, organizadores. Políticas, Planejamento e Gestão em Saúde - Abordagens e Métodos de Pesquisa. Rio de Janeiro: Editora Fiocruz; 2015. p. 81-111

3. Baptista TWF, Azevedo CS, Machado CV, organizadores. Políticas, Planejamento e Gestão em Saúde - Abordagens e Métodos de Pesquisa. Rio de Janeiro: Editora Fiocruz; 2015.

4. Minayo MCS, Gomes R, Almeida MF, Goldbaum M, Carvalheiro JR. A Abrasco faz Ciência e avança conhecimentos: contribuições da Ciência \& Saúde Coletiva e da Revista Brasileira de Epidemiologia. In: Lima NT, Santana JP, Paiva CHA, organizadores. Saúde Coletiva: a Abrasco em 35 anos de história. Rio de Janeiro: Editora Fiocruz; 2015. p. 101-114.

5. Pritchard A. Statistical bibliography or bibliometrics? Journal of Documentation 1969; 25(4):348-349.

6. Van Eck NJ, Waltman L. Software survey: VOSviewer, a computer program for bibliometric mapping. Scientometrics 2010; 84(2):523-538.

7. Van Eck NJ, Waltman L. How to normalize cooccurrence data? An analysis of some well-known similarity measures. J Am Soc Inf Sci Technol 2009; 60(8):16351651.

8. Paim J, Teixeira CF. Política, Planejamento e Gestão em Saúde: balanço do estado da arte. Rev Saude Publica 2006; 40(n. esp.):73-78.

9. Novaes HMD, Werneck GL, Cesse EAP, Goldbaum M, Minayo MCS. Pós-Graduação senso estrito em Saúde Coletiva e o Sistema Único de Saúde. Cien Saude Colet 2018; 23(6):2017-2025.

10. Minayo MCS, Gomes R. Ciência \& Saúde Coletiva no contexto nacional e internacional da divulgação científica. Cien Saude Colet 2015; 20(7):2013-2022

11. Machado CV, Faria TW, Nogueira CO. Políticas de saúde no Brasil nos anos 2000: a agenda federal de prioridades. Cad Saude Publica 2011; 27(3):521-532.

12. Viana ALD, Lima LD, organizadores. Regionalização e Relações Federativas na Política de Saúde no Brasil. Rio de Janeiro: Contra Capa; 2011.

13. Rivera FJU, Artmann E. Planejamento e Gestão em Saúde: conceitos, história e propostas. Rio de Janeiro: Ed. Fiocruz; 2020.

14. Teixeira CF. Planejamento em Saúde: conceitos, métodos, experiências. Salvador: Edufba; 2010. 
